\title{
Novedades en la distribución del género Ochthera (Diptera: Ephydridae) en Colombia
}

\section{Novelties on the distribution of the genus Ochthera (Diptera: Ephydridae) from Colombia}

\begin{abstract}
Javier O. Martínez-Alava ${ }^{1}$; Francisco Serna ${ }^{2}$
1'Ing. Agrónomo, M.Sc. en Ciencias Biológicas-Sistemática. Facultad de Ciencias Agrarias, Universidad Nacional de Colombia Museo Entomológico UNAB, Grupo Sistemática de Insectos Agronomía SIA, Bogotá, e-mail: jomartineza@unal.edu.co, Dhttps://orcid.org/0000-0002-4600-028X

${ }^{2}$ Ing. Agrónomo, M.Sc. en Entomología, Ph.D. Biological Sciences, Profesor Asociado, Facultad de Ciencias Agrarias. Universidad Nacional de Colombia, Museo Entomológico UNAB, Grupo Sistemática de Insectos Agronomía SIA, Bogotá, e-mail: fjsernac@unal.edu.co, (Dhttps://orcid.org/0000-0002-61439821
\end{abstract}

Cómo citar: Martínez-Alava, J.O.; Serna, F. 2019. Novedades en la distribución del género Ochthera (Diptera: Ephydridae) en Colombia. Rev. U.D.C.A Act. \& Div. Cient. 22(1):e1146. https://doi.org/10.31910/rudca.v22.n1.2019.1146

Artículo de acceso abierto publicado por Revista U.D.C.A Actualidad \& Divulgación Científica bajo una licencia Creative Commons CC BY-NC 4.0

Recibido: Junio 8 de 2018

Aceptado: Enero 9 de 2019

\section{RESUMEN}

Se actualiza la información taxonómica y geográfica de Ochthera para Colombia, un género con especies benéficas, reconocidas por sus hábitos depredadores de insectos pequeños terrestres y larvas de mosquitos. Se incluye el primer registro de Ochthera (O.) pilosa y nuevos datos de distribución para Ochthera $(O$.$) regalis y Ochthera (O$. angustifacies. Se agregan ilustraciones, comentarios de su distribución y mapas.

Palabras clave: angustifacies; pilosa; regalis; moscas de las riveras; taxonomía.

\section{ABSTRACT}

We update taxonomic and geographic information for genus Ochthera in Colombia, a taxon containing beneficial species known by their predatory habits on terrestrial insects and mosquito larvae. We consider a first record of Ochthera (O.) pilosa, and new distribution data of Ochthera $(O$.$) regalis and Ochthera (O.) angustifacies, adding$ illustrations, comments on their distribution, and maps.

Keywords: angustifacies; pilosa; regalis; shore flies; taxonomy.

\section{INTRODUCCIÓN}

Los Ephydridae, se conocen comúnmente como moscas de las riberas o moscas de los playones. Los adultos son de tamaño pequeño a mediano (longitud corporal 0,6-11,0mm); moscas usualmente opacas y oscuras (Figura 1), a menudo, con microtomento blancuzco, grisáceo o parduzco; rara vez, diversos en estructura corporal, vestidura y ornamentación; hembras generalmente más grandes que los machos. Cabeza grande; con una a tres, rara vez cinco, setas fronto-orbitales lateroclinadas o reclinadas/proclinadas; setas pseudopostocelares divergentes o ausentes. Arista pectinada solo dorsalmente, desnuda o pubescente. Facia plana a conspicuamente protuberante. Escuto usualmente con numerosas setulas acrosticales, una a cinco setas dorsocentrales, dos a tres setas supra-alares, una seta postalar; anepisterno setuloso. Ala comúnmente bien desarrollada, hialina, a veces maculada; $\mathrm{C}$ con quiebres humeral y Subcostal; Sc incompleta; R1 unida a la $\mathrm{C}$ en la mitad proximal del ala; celdas bm y dm confluentes, bm-cu ausente; celda cup ausente. Tibias anteriores y posteriores carecen de seta preapical dorsal (Mathis, 2010).

Esta familia agrupa 1.842 especies en 127 géneros de las subfamilias Discomyzinae, Hydrelliinae, Gymnomyzinae, Ilytheinae y Ephydrinae 
(Zatwarnicki, 1992). Para el Nuevo Mundo, se registran 743 especies en 86 géneros (Mathis, 2010). Para Colombia, se conocen 53 especies, pertenecientes a 26 géneros, 15 tribus y las cinco subfamilias (Mathis et al. 2016).

Dentro de los Ephydridae, los adultos de Ochthera, se distinguen por su tamaño mayor y coloración oscura, principalmente negra (Figura 1a, c, e); cabeza en vista lateral con o sin una protuberancia facial (carena) media distintiva, que se extiende ligeramente anterior al margen oral anterior; cara en vista anterior con o sin marcas distintivas (Figura 1b, d, g). Patas protorácicas de tipo raptorial (Figura 1a, c, e); fémur muy agrandado y con espinas en su margen flexora; tibia con espuela apical grande y fuerte; basitarso del macho, algunas veces, con estructura sensorial en el ápice externo de la margen flexora y, a veces, también con estructura sensorial en la base del segundo segmento tarsal (Figura 1f) (Clausen, 1977; 1980; Mathis, 2010).

Adultos y larvas del género Ochthera son depredadores de pequeños insectos terrestres y larvas de mosquitos (Simpson, 1975; Clausen, 1977; Mathis, 2010; Marshall, 2012); las larvas son depredadoras, principalmente de formas inmaduras de Chironomidae (Wirth et al. 1987).

El género Ocbthera pertenece a la tribu Ochtherini de la subfamilia Gymnomyzinae. Ochthera, se compone de dos subgéneros: Haplochila, caracterizado por un clípeo muy grande, triangular, largo y puntudo y Ochthera, con clípeo pequeño, subtriangular, no puntudo (Suh \& Kwon, 2009). El género es de distribución mundial (Clausen, 1977). De las 37 especies descritas en el subgénero Ochthera, Ochthera (O.) angustifacies (Hendel, 1930) y Ochthera $(O$.$) regalis Williston, 1897,$ cuentan con registros de su presencia en Colombia (Clausen, 1980; Mathis, 2010; Mathis et al. 2016); no obstante, hasta ahora no se conocían especímenes asociados a ninguna localidad en el país.

El objetivo del presente trabajo fue registrar datos taxonómicos y geográficos nuevos del género Ochthera (Diptera: Ephydridae) para Colombia, incluyendo, la presencia de una especie no conocida antes en el país.

\section{MATERIALES Y MÉTODOS}

Los acrónimos de las colecciones visitadas son: UNAB-Universidad Nacional, Agronomía, Bogotá, Museo entomológico de la Facultad de Ciencias Agrarias de la Universidad Nacional de Colombia; CTNI-Colección Taxonómica Nacional de Insectos "Luis María Maurillo"; MEFLG-Museo entomológico "Francisco Luis Gallego".

Las características morfológicas de los especímenes, se estudiaron a través de un estereomicroscopio NIKON SMZ-800. Para la identificación, a nivel de género, se utilizó la clave de Mathis (2010). Las identificaciones de las especies se adelantaron mediante el uso de las claves de Clausen $(1977 ; 1980)$.

En la lista de los especímenes examinados, se adiciona, entre corchetes, alguna información que no aparece en los rótulos originales de datos de colección, como el nombre de la cabecera municipal, coordenadas geográficas, altitud y la Colección depositaria, con su número de catálogo.

Las imágenes, se tomaron con una cámara SONY ILCE-5000, adaptada a un estereomicroscopio, mediante uno de los lentes oculares. Las imágenes digitales, se apilaron con el programa Helicon Focus ${ }^{\circledR}$ y se editaron con Adobe ${ }^{\circledR}$ Photoshop ${ }^{\circledR}$ CC versión 14,0.

Con el fin de representar la distribución geográfica de las especies para Colombia, a partir de las georreferencias, se produjeron mapas de los sitios de localización, según los datos consignados en los rótulos de recolección (las abreviaturas en los rótulos siguen a Martínez \& Serna (2015)), y mediante el programa de acceso libre en internet QGIS (QGIS Development Team, 2015).

\section{RESULTADOS Y DISCUSIÓN}

Se hallaron 23 especímenes del género Ochthera, pertenecientes a Ochthera (Ochthera) angustifacies $(\mathrm{n}=5)$, Ochthera (Ochthera) pilosa $(\mathrm{n}=$ 1) y Ochthera (Ochthera) regalis $(\mathrm{n}=17)$. Ochthera (Ochthera) pilosa se registra, por primera vez, para Colombia. Su localización, se muestra en la figura 2.

Ochthera (Ochthera) angustifacies (Hendel)

(Figs. 1a,b, 2)

Stenochthera angustifacies Hendel, 1930:132-133

Distribución. Sur de México, América Central y norte de Sudamérica: Argentina, Bolivia, Brasil, Colombia, Costa Rica, Ecuador, Guatemala, Honduras, Jamaica, México (Chiapas, Veracruz), Panamá, Paraguay, Perú (Clausen, 1980; Mathis et al. 2016).

Especímenes examinados. COLOMBIA: Guaviare: San José del Guaviare, Vereda Playa Güio, Reserva Puerto Amor, N 2³4'13.5”, W 72³8'45”, 175m, captura manual, 6-Mar-2018, K. Meneses, 1 을 [UNAB 4770]; datos iguales, trampa Malaise, 8-Mar-2018, C. Gómez, 1 ㅇ [UNAB 4770]; datos iguales, captura con jama, en planta de Bosque Húmedo Tropical, 6-Mar-2018, L. León, 1 + [UNAB 4770]; datos iguales, captura con jama, 8-Mar-2018, M. Cortes, 1 ; datos iguales, captura con jama en bosque de galería, 9-Mar-2018, K. Alzate, $10 \hat{\jmath}$ [UNAB 4770].

Comentarios. Ochthera (O.) angustifacies se cita para Colombia por Clausen (1980) y Mathis et al. (2016), sin indicar localidades.

\section{Ochthera (Ochthera) pilosa Cresson}

(Figuras 1c,d, 2)

Ochthera pilosa Cresson, 1926:255

Distribución. Sudeste de los Estados Unidos, México, América Central y el occidente de Sudamérica: Arizona, Nuevo México, Bolivia, Guatemala, México, Panamá y Perú (Clausen, 1977). 
Especímenes examinados. COLOMBIA: Cundinamarca: Tena, Vereda Catalamonte, Laguna de Pedro Palo, N 441'14.6", W 74²3’13.2”, 2019m, 20-Sep-1997, R. Paredes, 1 ㅇNAB 641].

Comentarios. Se registra, por primera vez, para Colombia, en el municipio de Tena (Cundinamarca).

\section{Ochthera (Ochthera) regalis Williston}

(Figuras 1e,g, 2)

Ochthera regalis Williston, 1897:6
Distribución. Sur de México, Indias Occidentales y norte de Sudamérica: Argentina, Belice, Bolivia, Brasil, Colombia, Costa Rica, República Dominicana, Ecuador, Guatemala, Guyana, Honduras, México (Chiapas, Guerrero, Nayarit, Oaxaca, Tabasco, Veracruz), Nicaragua, Panamá, Perú, Surinam, Trinidad, Venezuela, Indias Occidentales (Cuba, Gran Caimán, Jamaica, Puerto Rico) (Clausen, 1980; Mathis et al. 2016).

Especímenes examinados. COLOMBIA: Antioquia: Carepa, Fca. Tulenapa, N 7³9', W 76²6', 57m, Jama, 30-Mar-2014, A. Gamba, 1 ô (UNAB 893); Boyacá: Caldas, N 5³3'15”, W 7351'56”,

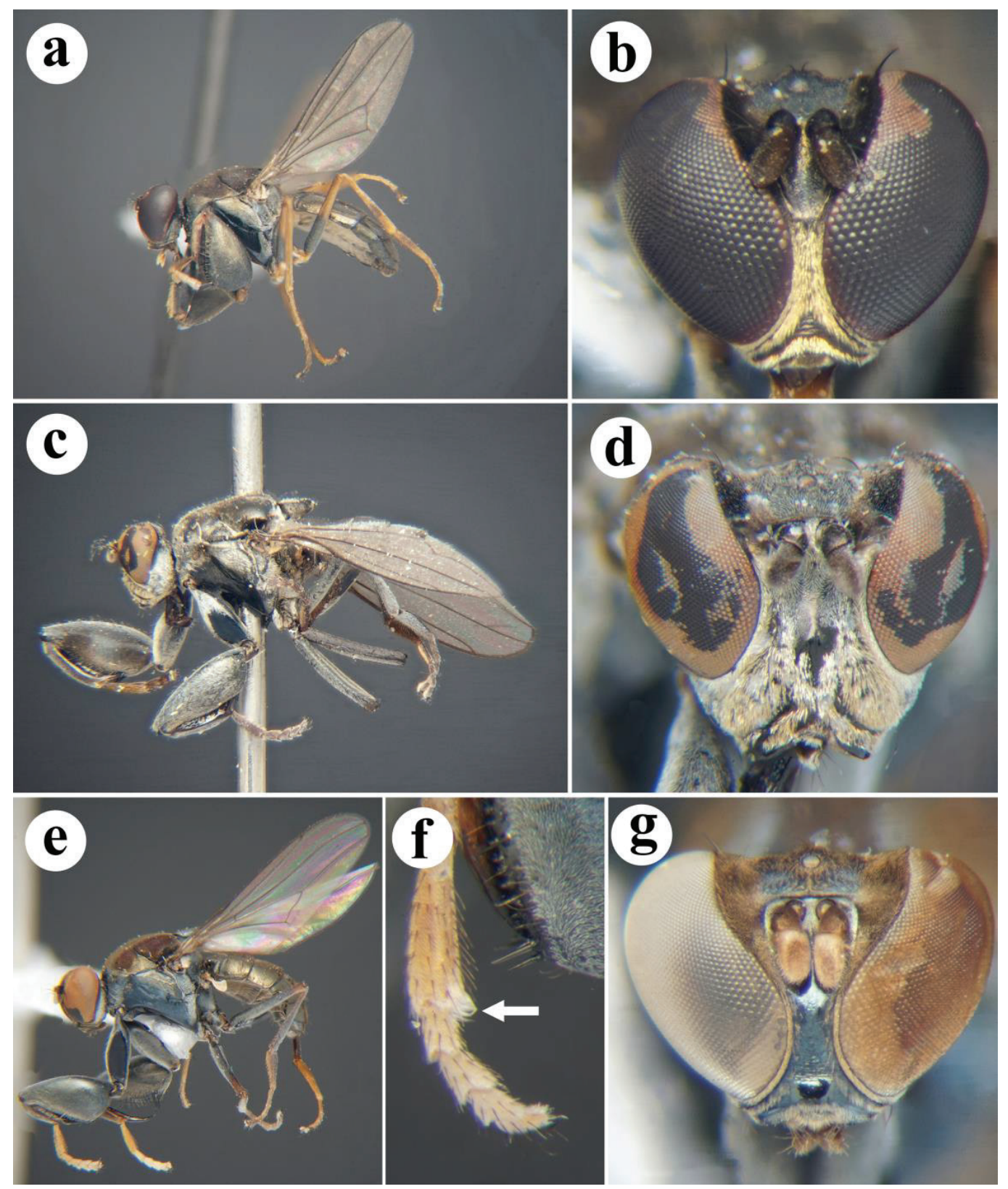

Figura 1. a) Habitus Ochthera (O.) angustifacies; b) Cabeza en vista anterior de Ochthera (O.) angustifacies; c) Habitus de Ochthera (O.) pilosa; d) Cabeza en vista anterior de Ochthera (O.) pilosa; e) Habitus Ochthera (O.) regalis; f) tarsómeros de la pata protorácica de Ochthera (O.) regalis (flecha indicando la estructura sensorial del basitarso); g) Cabeza en vista anterior de Ochthera (O.) regalis. 


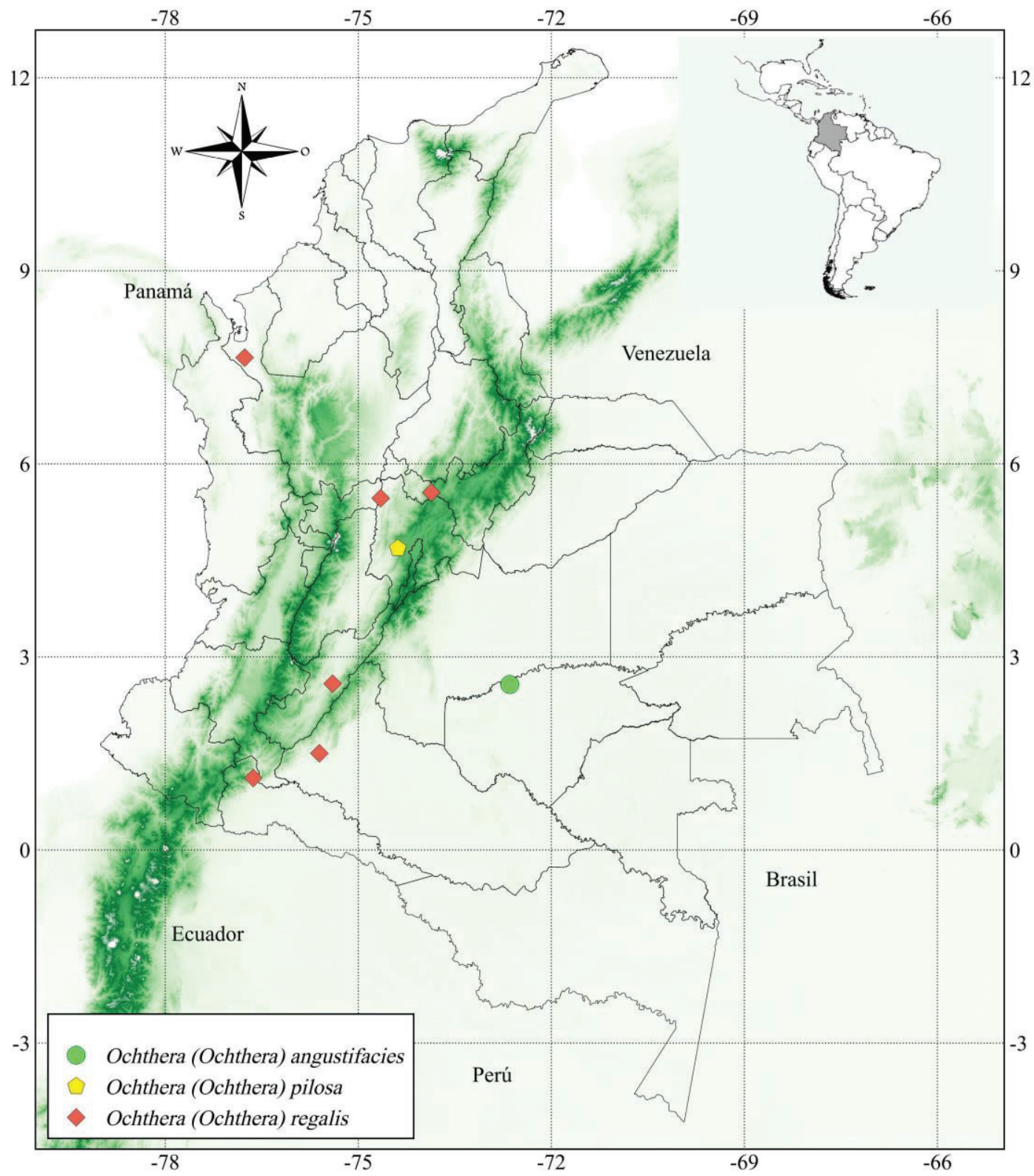

Figura 2. Localización de Ochthera (O.) angustifacies, Ochthera (O.) pilosa y Ochthera (O.) regalis en Colombia.

2680m, 1-Ene-2009, P. Osorio, 2 q [CTNI 193]; Caquetá: Florencia, CIMAZ-Macagual, N 1³0'3.99”, W 75³6’22.89”, 250m, 26-Mar2018, D. Bautista, $1 \widehat{\delta}^{\lambda}$ (UNAB 642); Cundinamarca: Puerto Salgar (C) [Cundinamarca], [N 5²7'55”, W 74³9’11”, 177m], [en] malezas, 31-I [Ene]-1959, G. Bravo, $2 \hat{\jmath}$ y 5 ㅇ (CTNI 193); datos iguales, 1 우 [MEFLG]; Huila: Campo Alegre, Vereda Los Rosales, N $2^{\circ} 35^{\prime}$, W 75²4', 607m, manual, 20-Mar-2015, M. Mendoza, 1 ㅇ [UNAB 642]; Putumayo: Mocoa, Vereda Caliyaco, Jardín Botánico Tropical Amazónico, N 107’06", W 76³7'58.7”, 580m, 18-Sep-2015, D. Bautista, $3 \hat{0}$ [UNAB 642]; datos iguales, $584 \mathrm{~m}$, manual, 18-Sep2015, V. Ramírez, 1 [ [UNAB 642]; datos iguales, N 17'6", W

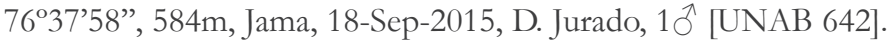
Comentarios. Ochthera $(O$.$) regalis contiene registros previos para$
Colombia, por Clausen (1980) y Mathis et al. (2016), sin indicar localidades.

Agradecimientos: Los autores agradecen especialmente al Museo Entomológico UNAB (Universidad Nacional Agronomía, Bogotá), por suministrar las herramientas necesarias para realizar la identificación de los ejemplares y elementos para realizar el proceso de curaduría. A COLCIENCIAS, por la financiación del doctorado del primer autor, en el marco del Programa de Formación Doctoral en Colombia. A Erika Valentina Vergara, por el préstamo de los especímenes de la Colección Taxonómica Nacional de Insectos "Luis María Murillo" (CTNI). Conflicto de intereses: El manuscrito fue preparado y revisado con participación de todos los autores, 
quienes declaramos que no existe conflicto de intereses que ponga en riesgo la validez de los resultados presentados.

\section{REFERENCIAS}

1. CLAUSEN, P.J. 1977. A revision of the Nearctic, Neotropical, and Palearctic species of the genus Ochthera including one Ethiopian species, and one new species from India. Transactions of the American Entomological Society (EE. UU.). 103(3):451-530.

2. CLAUSEN, P.J. 1980. Modifications to the genus Ochthera (Diptera: Ephydridae) and additions to the Neotropical species. Transactions of the American Entomological Society (EE. UU.). 106(2):205-222.

3. CRESSON, E.T. Jr. 1926. Description of new genera and species of (Ephydridae and Micropezidae). Transactions of the American Entomological Society (EE. UU.). 52(3):249274.

4. HENDEL, F. 1930. Die Ausbeute der deutschen ChacoExpedition 1925/26. Diptera. XIX. Ephydridae. Konowia (Austria). 9(2):127-155.

5. MARSHALL, S.A. 2012. Flies: the natural history and diversity of Diptera. Firefly Books. Richmond Hill, Canada. 616p. https://doi.org/10.1111/aen.12025

6. MARTÍNEZ A., J.O.; SERNA, F. 2015. Managing insect collections. Micropezidae (Diptera: Nerioidea) of the Entomological Museum UNAB. Agronomía Colombiana (Colombia). 33(3):339-347. http://dx.doi.org/10.15446/ agron.colomb.v33n3.52432

7. MATHIS, W.N. 2010. Ephydridae (Shore flies). En: Brown, B.V.; Borkent, A.; Cumming, J.M.; Wood, D.M.; Woodley, N.E.; Zumbado, M. (eds). Manual of Central American Diptera.
Vol. 2. NRC Research Press, Ottawa. p.1211-1233. https:// doi.org/10.1899/30.3.BR.868

8. MATHIS, W.N.; RODRIGUES, F. DE A.; COURI, M.S. 2016. Family Ephydridae. Zootaxa (Nueva Zelanda). 4122(1):752770. https://doi.org/10.11646/zootaxa.4122.1.64

9. QGIS. Development team. 2015. GISA Free and Open Source Geographic Information System.

10. SIMPSON, K.W. 1975. Biology and immature stages of three species of Nearctic Ochthera (Diptera: Ephydridae). Proceedings of the Entomological Society of Washington (EE. UU.). 77(1):129-155

11. SUH, S.J.; KWON, Y.J. 2009. First record of the genus Ocbthera Latreille (Diptera: Ephydridae) in Korea. Animal Cells and Systems (Corea del Sur), 13(4):461-464. https://doi.org/ 10.1080/19768354.2009.9647242

12. WIRTH, W.W.; MATHIS, W.N.; VOCKEROTH, J.R. 1987. Ephydridae En: McAlpine, J.F.; Peterson, B.V.; Shewell, G.E.; Teskey, H.J.; Vockeroth, J.R.; Wood D.M. (eds). Manual of Nearctic Diptera. vol. 2. Monograph of the Biosystematics Research Institute, No. 28. Agriculture Canada, Ottawa. p.1027-1047.

13. WILLISTON, S.W. 1897. Diptera Brasiliana. Part IV. Kansas University Quarterly (EE. UU.), Series A. 6:1-12

14. ZATWARNICKI, T. 1992. A new classification of Ephydridae based on phylogenetic reconstruction (Diptera: Cyclorrhapha). Genus (Italia). 3(2):65-119. 